\title{
The CAR mRNA-Interaction Surface is a Zipper Extension of the Ribosome A Site
}

\author{
Carol Dalgarno ${ }^{1}$, Kristen Scopino ${ }^{1}$, Mitsu Raval ${ }^{1}$, Clara Nachmanoff ${ }^{1}$, Eric Sakkas ${ }^{1}$, Daniel Krizanc \\ 2,3, Kelly M. Thayer ${ }^{2,3,4}$ and Michael P. Weir 1,3,* \\ 1 Department of Biology, Wesleyan University, Middletown, CT 06459, USA; cdalgarno@wesleyan.edu (C.D.); \\ kscopino@wesleyan.edu (K.S.); mraval@wesleyan.edu (M.R.); cnachmanoff@wesleyan.edu (C.N.); \\ esakkas@wesleyan.edu (E.S.) \\ 2 Department of Mathematics and Computer Science, Wesleyan University, Middletown, CT 06459, USA; \\ dkrizanc@wesleyan.edu (D.K.); kthayer@wesleyan.edu (K.S.) \\ 3 College of Integrative Sciences, Wesleyan University, Middletown, CT 06459, USA \\ 4 Department of Chemistry, Wesleyan University, Middletown, CT 06459, USA \\ * Correspondence: mweir@wesleyan.edu
}

Keywords: ribosome translocation, molecular dynamics, CAR interaction surface, A-site decoding center, ribosome substates

\begin{abstract}
The ribosome CAR interaction surface behaves like an extension of the decoding center $A$ site and has $\mathrm{H}$-bond interactions with the +1 codon that is next in line to enter the $A$ site. Through molecular dynamics simulations, we investigated the codon sequence specificity of this CARmRNA interaction and discovered a strong preference for GCN codons, suggesting that there may be a sequence-dependent layer of translational regulation dependent on the CAR interaction surface. Dissection of the CAR-mRNA interaction through nucleotide substitution experiments showed that the first nucleotide of the +1 codon dominates over the second nucleotide position, consistent with an energetically favorable zipper-like activity that emanates from the A site through the CAR-mRNA interface. The +1 codon/CAR interaction is also affected by the identity of nucleotide 3 of $+1 \mathrm{GCN}$ codons which influences the stacking of $\mathrm{G}$ and $\mathrm{C}$. Clustering analysis suggests that the $A$ site decoding center adopts different neighborhood substates that depend on the identity of the +1 codon.
\end{abstract}

\section{Introduction}

Protein translation is controlled by multiple mechanisms providing overlapping layers of regulation that operate at the levels of translation initiation, elongation, and termination. Phosphorylation of elF2 and eEF2 regulate initiation and elongation respectively [1-3] and regulated modifications of tRNA, rRNA and mRNA nucleotides can also affect these translation stages and influence translation fidelity and efficiency [4-9]. Interestingly, K63 polyubiquitination of solvent exposed ribosome proteins can halt translation $[10,11]$ and translation is also modulated through ribosome biogenesis [12]. In recent years, increasing evidence has emerged suggesting that alternative versions of ribosomes with different translation properties are made under different cellular conditions such as stress [13-16]. Alternative versions of ribosomes arise through several mechanisms: they may lack or have different isoforms of ribosomal proteins, or they can have differentially regulated residue modifications in their ribosomal proteins or rRNAs. Integrating these observations with our structural understanding of ribosome function presents an exciting challenge 

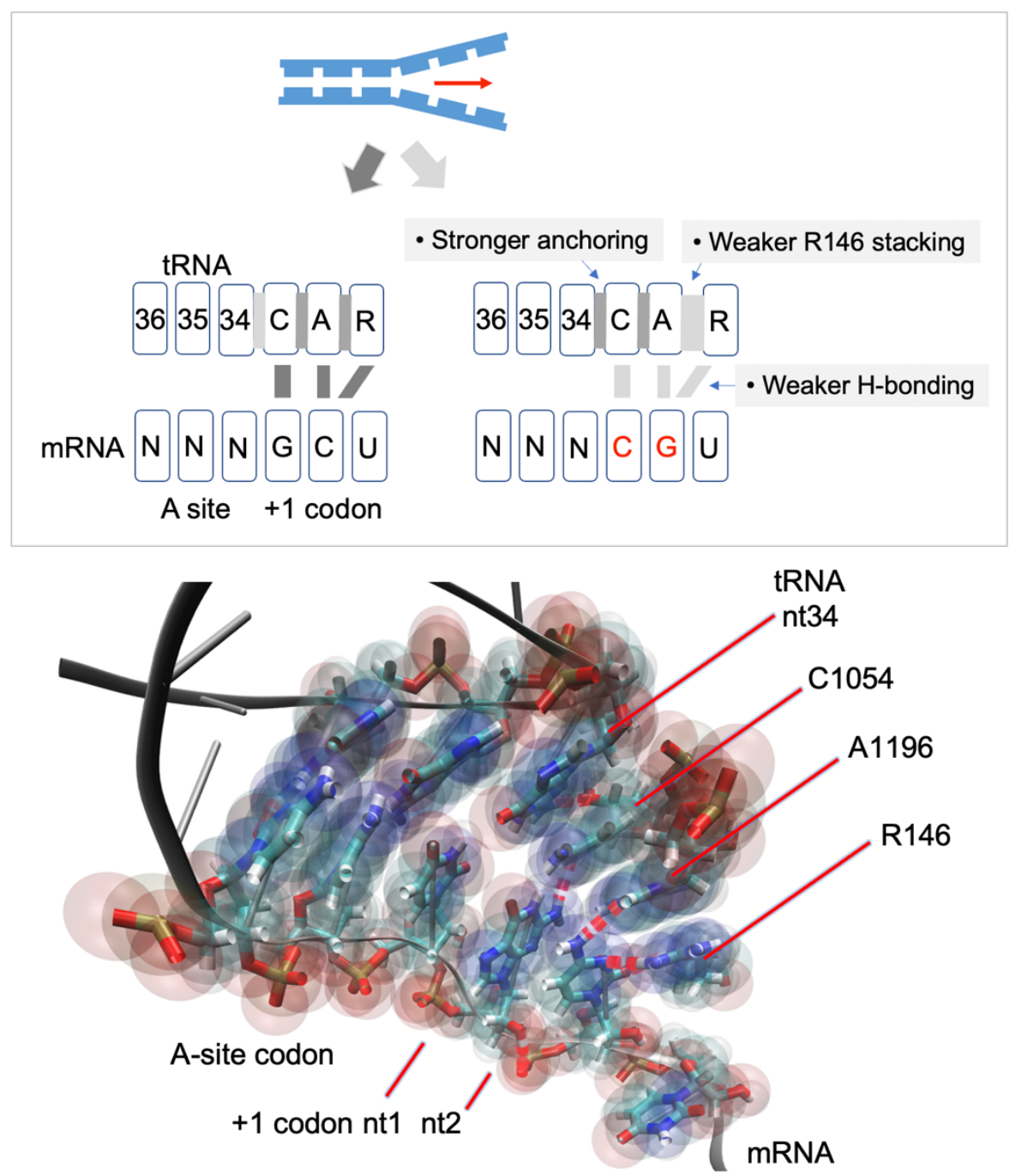

Figure 1. The ribosome CAR surface interacts with the mRNA +1 codon. The CAR surface is positioned close to the mRNA +1 codon allowing for $\mathrm{H}$-bonding between CAR and the codon. Our study shows that this interaction is strongest with the $+1 \mathrm{GCU}$ codon. The weaker interaction with other +1 codons is associated with weaker stacking interactions between A1196 and R146, and stronger anchoring through stacking between C1054 and tRNA nt 34. The CAR/+1 codon interaction behaves like a zipper (see text).

Using molecular dynamics (MD) methods, we recently identified a ribosome interaction surface termed CAR (Fig 1) that we hypothesize provides a layer of translation regulation under stress conditions through production of ribosomes with an altered version of the CAR surface [17-19]. Protein translation requires efficient translocation of mRNA codons through the decoding center aminoacyl (A) site where the codon base pairs with the anticodon of the tRNA that brings in the next amino acid for the growing newly synthesized protein. We hypothesize that interaction of CAR with the mRNA helps modulate this translocation. The CAR interaction surface consists of three residues: nucleotides $\mathrm{C} 1274$ and $\mathrm{A} 1427$ of the $S$. cerevisiae $18 \mathrm{~S}$ rRNA-which we will refer to as C1054 and A1196, the corresponding nucleotides in E. coli 16S rRNA-and R146 of ribosomal protein Rps3 [19]. C1054 and A1196 are well conserved in 18S and 16S rRNAs, but $\mathrm{R} 146$ is only conserved in eukaryote ribosomes, suggesting that prokaryotes may have a partial version of the $C A(R)$ surface [19]. Integrity of the CAR interface relies on stacking interactions, namely base stacking between C1054 and A1196, and pi-cation stacking between the A1196 base and the guanidinium group of R146. The CAR surface looks like an extension of the A-site 
tRNA anticodon (Fig 1) and is anchored to the anticodon through a base stacking interaction between C1054 and tRNA nucleotide 34, the anticodon nucleotide that base pairs to the wobble nucleotide of the codon.

Immediately adjacent to the A-site tRNA anticodon, the CAR surface is positioned facing the +1 codon of the mRNA - the codon that is next in line to enter the ribosome A site-allowing the CAR surface to have sequence-dependent $\mathrm{H}$-bond interactions with the +1 codon nucleotides (Fig 1). When the +1 codon is GCU, the first base (+1 G1) base pairs with $\mathrm{C} 1054$ through Watson-Crick edges of both bases, and the second base (+1 C2) H-bonds with A1196 and R146; +1 C2 interacts with A1196 through a Watson-Crick/Hoogsteen interaction and R146 through $\mathrm{H}$-bonding with the planar guanidinium group of the arginine. The interaction between CAR and the +1 codon evolves dynamically during the various stages of ribosome elongation [20] and is most pronounced at translocation stage II [19], the stage analyzed in the current study.

$\mathrm{R} 146$ is post-translationally modified through methylation of its guanidinium group which causes the $\mathrm{H}$-bonding interaction between CAR and the +1 codon to be significantly reduced [18]. We hypothesize that ribosomes made under stress conditions have a CAR interface with an unmethylated R146 thereby potentiating the interaction between CAR and the +1 codon and permitting CAR-mediated regulation of translation [18]. Consistent with this model, the expression levels of Sfm1, the methyltransferase enzyme responsible for methylating R146 $[21,22]$, are downregulated under stress conditions [18,23], although this may be due in part to the depression of translation levels observed under stress [24].

We propose that energetically favorable interactions between CAR and the +1 codon are highly sequence specific, implying that CAR regulation of translation depends on the codon content of open reading frames (ORFs). Indeed, highly expressed genes have particularly high levels of GCN codons in the initial codons of their ORFs (ramp regions; [25]) and it has been suggested that codons in the ramp region might be particularly influential in controlling translation rates $[17,25]$ perhaps analogous to cars accelerating onto the on-ramp of a highway. In our initial studies, we investigated the effects of changing the second nucleotide of +1 codons and showed that the CAR/+1 codon interaction is strongest with GCU codons [19]. In this study, we have expanded this analysis and found that $+1 \mathrm{GCU}$ codons have the highest levels of $\mathrm{H}$ bonding with $\mathrm{CAR}$, and that the nucleotide at position 1 of the +1 codon is more influential than position 2 in determining the strength of the CAR/mRNA interaction, like a zipper extension of the A-site codon/anticodon base pairing. In addition, the nucleotide identity of position 3 modulates stacking of the other two +1 codon nucleotides and their interaction with CAR. Our structural analysis of the A-site neighborhood suggests that, in each of its codon steps during ribosome translocation, the decoding center neighborhood adopts different substates depending on the identity of the +1 codon. The sequence-dependent modulation of CAR/mRNA interactions may impact translation speeds and protein production levels in response to stress conditions.

\section{Results and Discussion}

Sequence specific interactions of CAR with the +1 codon, the codon next to enter the ribosome A site, are likely to be important for regulation of protein translation. To investigate energetically favorable interactions, we developed an information theoretic framework to compare different +1 codons and assess the relative importance of different nucleotide positions in the codon.

\section{A ramp codon position weight matrix reveals nucleotide selection preferences}

Individual information scores [26] of codons were used to guide our assessment of the behaviors of different codons. Individual information scores, which can reflect the free energies of a 
sequence motif's ligand interactions [27], are calculated based on the deviations of nucleotide frequencies from their expected background frequencies. Sequence patterns that have higher than expected frequencies may have selective advantages that often reflect biological functions, and patterns with lower than expected frequencies may have selective disadvantages. We hypothesized that these selective pressures on codons might result in part from interactions of +1 codons with the CAR interface, although of course many other codon properties such as tRNA availability [28] might also contribute to differences in selective pressure.

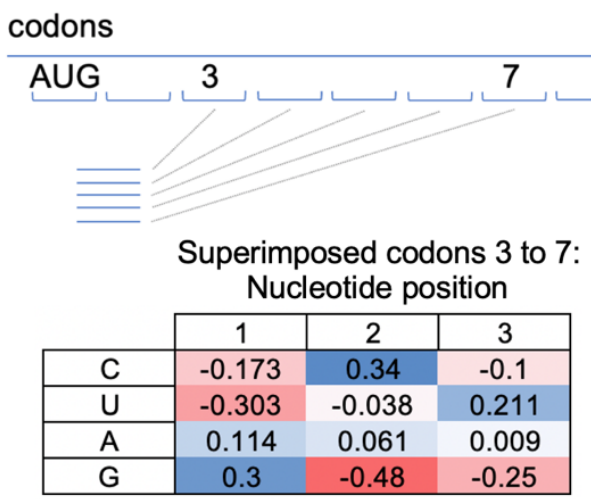

A. Position weight matrix

\begin{tabular}{|c|c|c|c|c|c|}
\hline & $\mathrm{C} 2$ & U2 & A2 & G2 & \\
\hline \multirow{4}{*}{ C1 } & 0.377 & -0.001 & 0.098 & -0.443 & U3 \\
\hline & 0.066 & -0.311 & -0.213 & -0.753 & C3 \\
\hline & 0.175 & -0.203 & -0.104 & -0.645 & A3 \\
\hline & -0.084 & -0.462 & -0.363 & -0.904 & G3 \\
\hline \multirow{4}{*}{ U1 } & 0.247 & -0.131 & -0.032 & -0.573 & U3 \\
\hline & -0.064 & -0.441 & -0.342 & -0.883 & C3 \\
\hline & 0.045 & -0.333 & -0.234 & -0.775 & A3 \\
\hline & -0.214 & -0.592 & -0.493 & -1.034 & G3 \\
\hline \multirow{4}{*}{ A1 } & 0.664 & 0.286 & 0.385 & -0.156 & U3 \\
\hline & 0.353 & -0.024 & 0.074 & -0.466 & $\mathrm{C} 3$ \\
\hline & 0.462 & 0.084 & 0.183 & -0.358 & A3 \\
\hline & 0.203 & -0.175 & -0.076 & -0.617 & G3 \\
\hline \multirow{4}{*}{ G1 } & 0.85 & 0.473 & 0.572 & 0.031 & U3 \\
\hline & 0.54 & 0.162 & 0.261 & -0.28 & C3 \\
\hline & 0.648 & 0.271 & 0.369 & -0.171 & A3 \\
\hline & 0.389 & 0.012 & 0.11 & -0.43 & G3 \\
\hline
\end{tabular}

B. Individual information of codons 3-7

\begin{tabular}{|c|c|c|c|c|c|}
\cline { 2 - 5 } \multicolumn{1}{c|}{} & C2 & U2 & A2 & G2 & \multicolumn{1}{c|}{} \\
\cline { 1 - 3 } C1 & CCU & CUU & CAU & CGU & \\
\cline { 1 - 3 } U1 & UCU & UUU & UAU & UGU & \multirow{2}{*}{ U3 } \\
\cline { 1 - 3 } A1 & ACU & AUU & AAU & AGU & \\
\cline { 1 - 3 } G1 & GCU & GUU & GAU & GGU & \\
\hline
\end{tabular}

C. Individual information of codons 3-7: tested NNU

Figure 2. Information theoretic analysis of codons. (A) Codons 3-7 of ORFs of 6033 yeast genes were superimposed to create a meta-codon representation. The position weight matrix shows $\log _{2}\left(f_{\text {observed }} / \mathrm{f}_{\text {expected }}\right)$ for nucleotides at each codon position based on expected background frequencies in ORFs (A: 0.328, C: 0.192, G: 0.204, U: 0.276). (B) Individual information scores for codons were calculated using the position weight matrix. For example, the score for GCU is $0.30+0.34+0.21=$ 0.85 . (C) NNU codons analyzed in this study with a heatmap color scale indicating their relative individual information scores. 
We computed a position weight matrix to score the individual information of codons in the ramp regions of ORFs (Fig 2A). We focused on codons 3-7 of ORFs (6033 verified and uncharacterized $S$. cerevisiae genes; https://www.yeastgenome.org) since these codons exhibit particularly strong GCN periodicity in highly expressed genes $[17,29,30]$ and we suspect this ramp region may be particularly influential in the hypothesized CAR-mediated regulation of translation $[17,19]$. By superimposing codons 3-7, we created a meta-codon representation of these codons examining nucleotide choices at the $1^{\text {st }}, 2^{\text {nd }}$, and $3^{\text {rd }}$ positions of each meta-codon. Each of the three nucleotide positions were treated as independent and the codon position weights were calculated using the background frequencies in ORFs (A: 0.328, C: 0.192, G: $0.204, \mathrm{~T}: 0.276)$. Addition of the position weights, $\log _{2}\left(\mathrm{f}_{\text {observed }} / \mathrm{f}_{\text {expected }}\right)$ across positions 1,2 and 3 of each codon sequence provided individual information scores (Fig 2B).

Since individual information scores reflect deviations from expected frequencies with potential functional correlates, we were interested in how trends in individual information scores might relate to CAR function measured by monitoring $\mathrm{H}$-bonding of CAR to +1 codon as well as the structural integrity of CAR. Comparisons of codon scores (Fig 2B) showed that GCU has the highest individual information, indicating the highest positive deviation from expected frequencies. We hypothesize that this may be related in part to CAR function given that the +1 GCU structure aligns with and exhibits significant $\mathrm{H}$-bonding to CAR predominantly through +1 G1-C1054 and +1 C2-A1196/R146. We were curious to compare CAR function for +1 codons over a range of individual information scores. Our previous testing [19], starting with $+1 \mathrm{GCU}$ and varying position 2 , showed that $+1 \mathrm{GCU}$ has the highest $\mathrm{H}$-bonding and $+1 \mathrm{GGU}$ has the lowest, similar to the individual information trend. We wished to expand this analysis to compare $A$ and $G$ at position 1 ( +1 GNN cf. +1 ANN) since these codons have similar individual information but might have different CAR behaviors. Similarly, we wished to find out whether +1 codons starting with $\mathrm{U}$ or $\mathrm{C}(+1 \mathrm{UNN}$ and $+1 \mathrm{CNN})$ have characteristic CAR behaviors given that their individual information scores tend to be lower and potentially detrimental. We decided to limit our initial analysis to +1 codons with position 3 fixed at $U$ (NNU, Fig 2C) given that our previous testing [19] suggested that positions 1 and 2 are likely to be the dominant interactors with CAR (Fig 1).

\section{CAR/mRNA H-bonding is sequence dependent}

MD simulations with the AMBER suite [31] were used to assess CAR interactions with the +1 codon as described previously $[18,19]$. We used a 494-residue subsystem of the ribosome centered around C1054 of the CAR interface that included the A-site codon and +1 codon of the mRNA, and an anticodon region of the tRNA. The residues at the edges of the subsystem were restrained to their initial coordinates (onion shell; $[18,19]$ ) to conform to translocation stage II (PDB ID 5JUP; [20]), the stage that exhibits the most robust CAR/+1 codon $\mathrm{H}$-bond interactions.

As discussed above, our previous analysis [19] showed that the $+1 \mathrm{GCU}$ codon interacts with the CAR interface dominated by $\mathrm{H}$-bond interactions between $+1 \mathrm{G} 1$ and $\mathrm{C} 1054$ of $\mathrm{CAR}$, and +1 C2 with A1196 and R146 of CAR. Minor interactions were also observed between +1 G1 and A1196 as well as $+1 \mathrm{G} 1$ with nt34 of the tRNA which normally $\mathrm{H}$-bonds with the A-site wobble nucleotide; +1 C2 had minor interactions with $\mathrm{C} 1054$. To ensure detection of major, minor and cross-registration interactions, we measured $\mathrm{H}$-bonding of A-site codon nt2 to tRNA nucleotides 36, 35 and 34; A-site nt3 to tRNA 35, 34 and C1054; +1 codon nt1 to tRNA nt34, C1054, and A1196; and +1 codon nt2 to C1054, A1196, and R146 for different +1 codons (Fig 3).

Our overall results showed that $+1 \mathrm{GCU}$ has the highest $\mathrm{H}$-bonding to CAR compared to the other tested +1 codons (Figs 1, 3 and S2). CAR H-bond levels are lower when +1 G1 or C2 are replaced, and these reductions are associated with elevated $\mathrm{H}$-bonding of the A-site wobble nucleotide with tRNA nt34. Replacement of $+1 \mathrm{G} 1$ or $\mathrm{C} 2$ also affects anchoring of CAR to the tRNA and stacking interactions of the CAR interface as discussed later. 
A

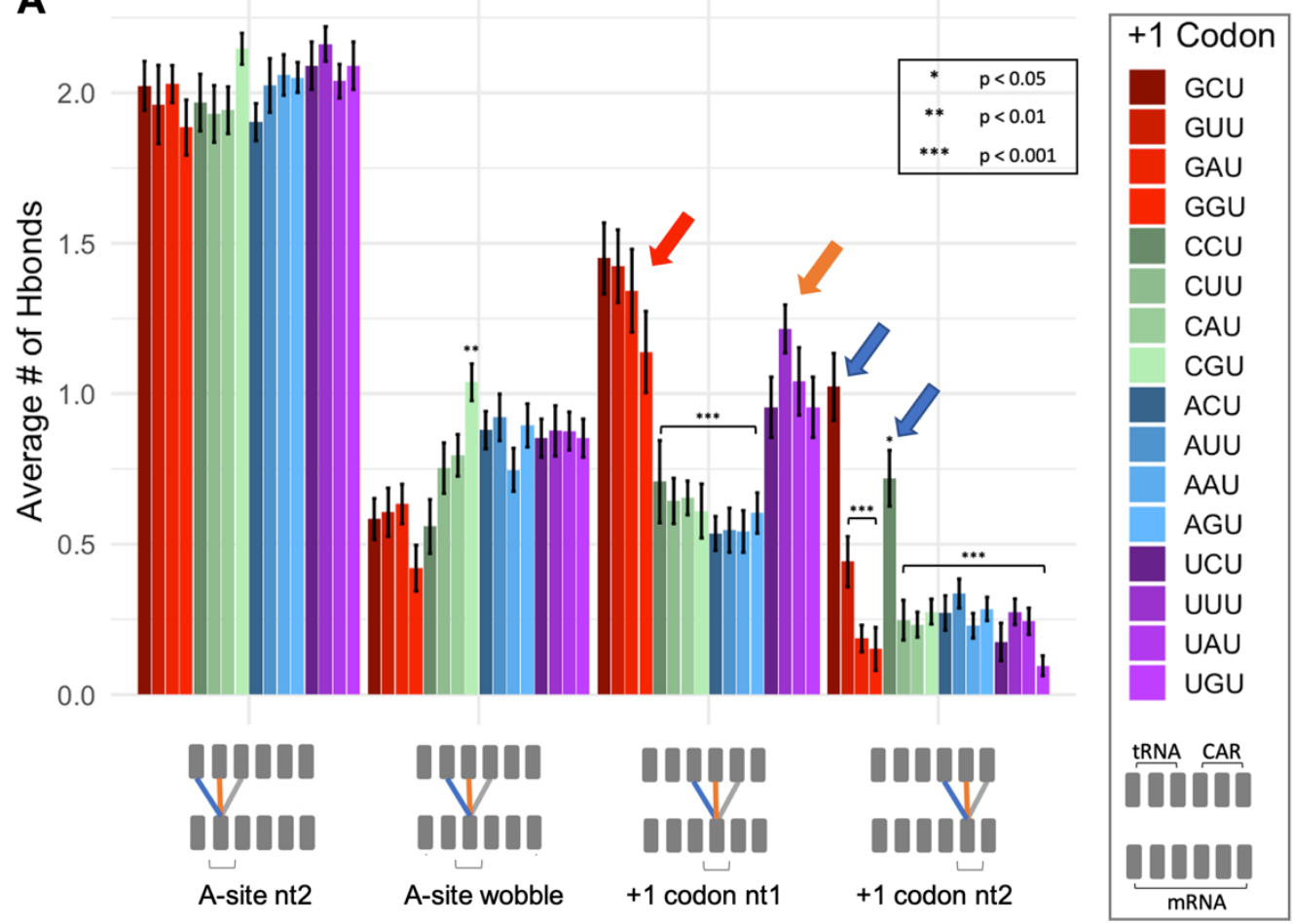

B

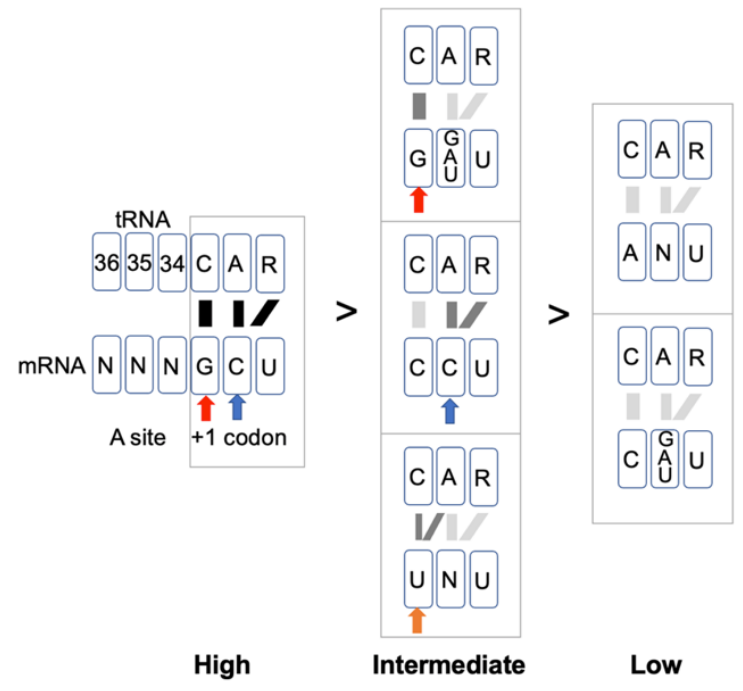

Figure 3. H-bond interactions of CAR. (A) Illustrated are H-bond counts between mRNA nucleotides (in the $A$ site and +1 codon next to enter the $A$ site) and the tRNA anticodon and ribosome CAR interface. Statistical tests show comparisons with $+1 \mathrm{GCU}$. Error bars show standard errors in $\mathrm{H}$-bond counts from the MD. (B) Groupings of +1 codons with significantly different levels of $\mathrm{H}$-bonding (t-test $\mathrm{p}<0.001$ ). +1 codons with $\mathrm{G}$ at position 1 (red arrows) or $\mathrm{C}$ at position 2 (blue arrows) have strong interactions. With $\mathrm{U}$ at position 1 (orange arrows) the $\mathrm{H}$-bonding is split between $\mathrm{C} 1054$ and $\mathrm{A} 1196$ (see Fig 4).

The levels of $\mathrm{H}$-bond interactions of the +1 codon with $\mathrm{CAR}$ can be grouped into a tiered series (Fig 3B): high (GCU), intermediate (G[GAU]U, CCU, UNU), and low (ANU, C[GAU]U).

The high group has optimal nucleotides at both positions 1 and 2 of the +1 codon $(+1 \mathrm{G} 1$ and $+1 \mathrm{C} 2)$; the intermediate group has optimal nucleotides at one of the two positions $(+1 \mathrm{G} 1$ or +1 C2) or UGN as discussed below; the low group has suboptimal nucleotides at positions 1 and 2 (not +1 G1 and not +1 C2). 
The UNU codons are qualitatively different from the other intermediate group codons as the +1 U1 interaction is split evenly between C1054 and A1196 (Fig 4, Fig S2). The prominent offphase U1/A1196 may be associated with properties that are selected against (see below). In contrast, $+1 \mathrm{G} 1$ shows a very strong $\mathrm{H}$-bonding preference for $\mathrm{C} 1054$, whereas $+1 \mathrm{C} 1$ and +1 A1 $\mathrm{H}$-bond at similarly low levels to the three residues: tRNA nt34, C1054 and A1196 (Fig 4).

Overall, these results suggest that the ribosome's CAR surface has sequence specific interactions with codons of the mRNA ORF when they are in the +1 position.

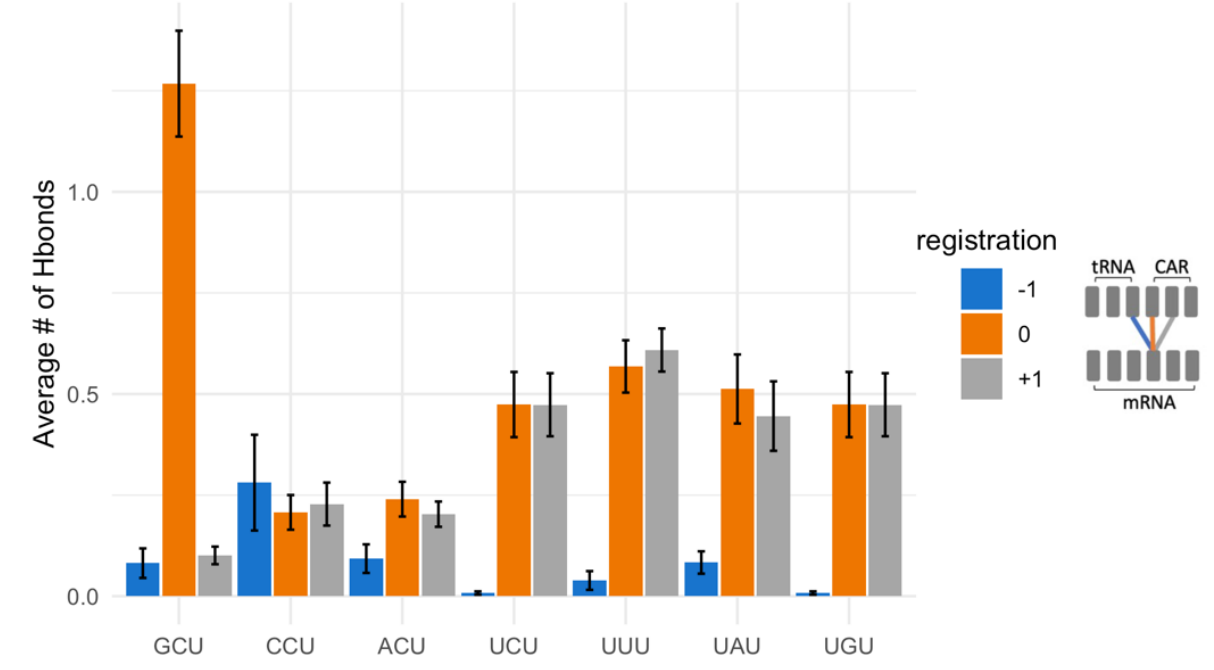

Figure 4. Alignment of CAR mRNA interactions. Nucleotide 1 of the $+1 \mathrm{GCU}$ codon has dominant $\mathrm{H}$-bond interactions with $\mathrm{C} 1054$. In contrast, the weaker interactions of $U$ at position $1(+1 \mathrm{UNN})$ are split between $\mathrm{C} 1054$ (orange) and A1196 (grey). C or A at position 1 have weak interactions with tRNA nt 34 (blue), C1054 and A1196.

\section{CAR behaves like an extension of the A-site anticodon}

Nucleotide substitutions of the optimal $+1 \mathrm{GCU}$ result in reduced $\mathrm{H}$-bonding between $\mathrm{CAR}$ and the +1 codon. These reductions show a graded effect from $5^{\prime}$ to $3^{\prime}$ in the +1 codon, suggesting a zipper-like behavior. If the $+1 \mathrm{G} 1$ interaction with $\mathrm{CAR}$ is disrupted by replacing $\mathrm{G} 1$, this leads to significant reductions in $\mathrm{H}$-bonding of both positions 1 and 2 of the +1 codon (Fig $5 A$ ). However, if the $+1 \mathrm{C} 2$ interaction is disrupted, this results in significant $\mathrm{H}$-bond reduction of only position 2 whereas position 1 has only minor reductions (Fig 5B). This suggests that interactions between the +1 codon nucleotides and CAR are facilitated by interactions on their 5 ' side suggesting a zipper-like effect emanating from the A site through the CAR/+1 codon interaction (Fig 1).

Although nucleotide 3 of the +1 codon does not interact directly with the CAR interface, replacing $\mathrm{U} 3$ in $+1 \mathrm{GCU}$ reduces $\mathrm{C} 2 \mathrm{H}$-bonding with $\mathrm{CAR}$ (Fig $5 \mathrm{C}$ ) suggesting that the third nucleotide of the +1 codon can influence the positioning of $\mathrm{C} 2$. Consistent with this, examination of G1:C2 base stacking (Fig 5D, Fig S3) showed that the alignment of the stacked base rings was less centered and more variable when U3 was replaced with other nucleotides.

Interestingly, codons with $U$ at position 3 have higher individual information scores than other third position nucleotides (Fig 2B). 

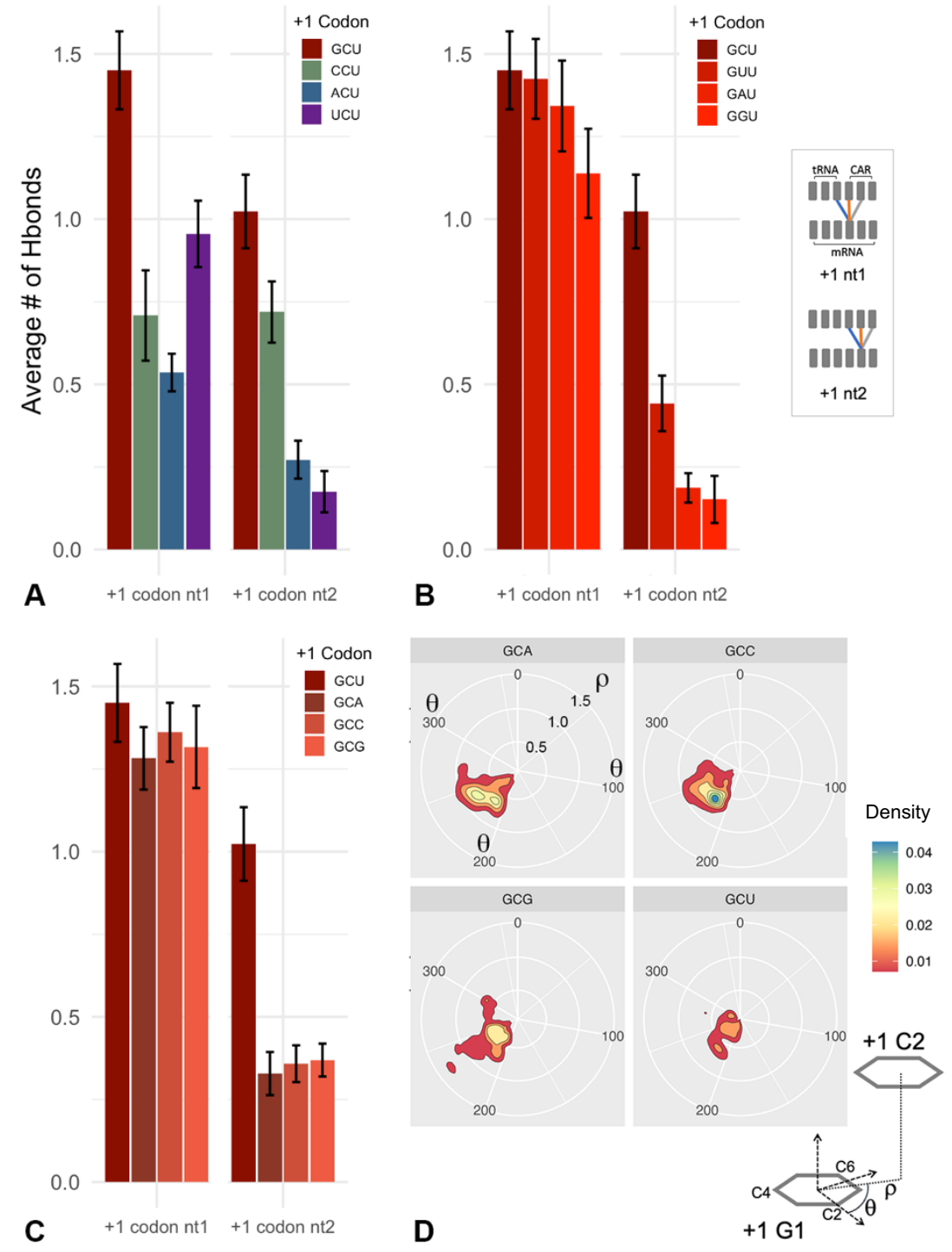

Figure 5. Influences of nucleotide positions on the CAR/+1 codon interaction. (A) When position 1 of the optimal $+1 \mathrm{GCU}$ codon is changed, both positions 1 and 2 of the codon have reduced $\mathrm{H}$ bonding with CAR and tRNA nt34. (B) When position 2 of +1 GCU is changed, position 2 has significantly reduced $\mathrm{H}$-bonding, but position 1 has minor reductions suggesting that the +1 codon interaction with CAR has zipper-like properties emanating from the A site. (C) When position 3 of +1 GCU is changed, the position 2 interaction with CAR is disrupted suggesting that position 3 may affect G1:C2 stacking. (D) A polar coordinate representation of G1:C2 stacking [32] across trajectories (4000 frames over $2000 \mathrm{~ns}$ for each +1 codon) shows that G1:C2 stacking is less centered (smaller $\rho$ values) and more variable when U3 is replaced with A3, C3 or G3. Note that smaller $\rho$ values closer to the center have less area due to the polar coordinate projection onto a plane (see Fig S3).

The above analysis suggests dominance of position 1 over position 2 in determining the extent of $\mathrm{H}$-bonding of CAR to the +1 codon. Hence, to compare the CAR interactions of codons with different individual information scores, we grouped our data according to which nucleotide was at position 1. Consistent with the apparent zipper behavior of the CAR/+1 codon interaction, we observe a correlation of codon individual information with the strength of the CAR/+1 codon interaction but only with optimal nucleotide choices at position 1 of the +1 codon ( $\mathrm{G} 1$ and to some extent $\mathrm{C} 1$; Fig 6B). For this analysis, we compared individual information scores of codons with the sum of the $\mathrm{H}$-bond interactions of nucleotides 1 and 2 with CAR (and nt 34; Fig $6 \mathrm{~A})$. We found that with $\mathrm{G} 1(+1 \mathrm{GNU})$ or $\mathrm{C} 1(+1 \mathrm{CNU})$, there is a correlation between the level 
of $\mathrm{H}$-bonding and individual information scores of codons (correlation coefficient $=0.818$; Fig $6 \mathrm{~B}$ ). Elevated individual information scores reflect selection away from expected background frequencies, and improved $\mathrm{CAR} /+1$ codon $\mathrm{H}$-bonding may contribute in part to this selection suggesting possible benefits of CAR function.

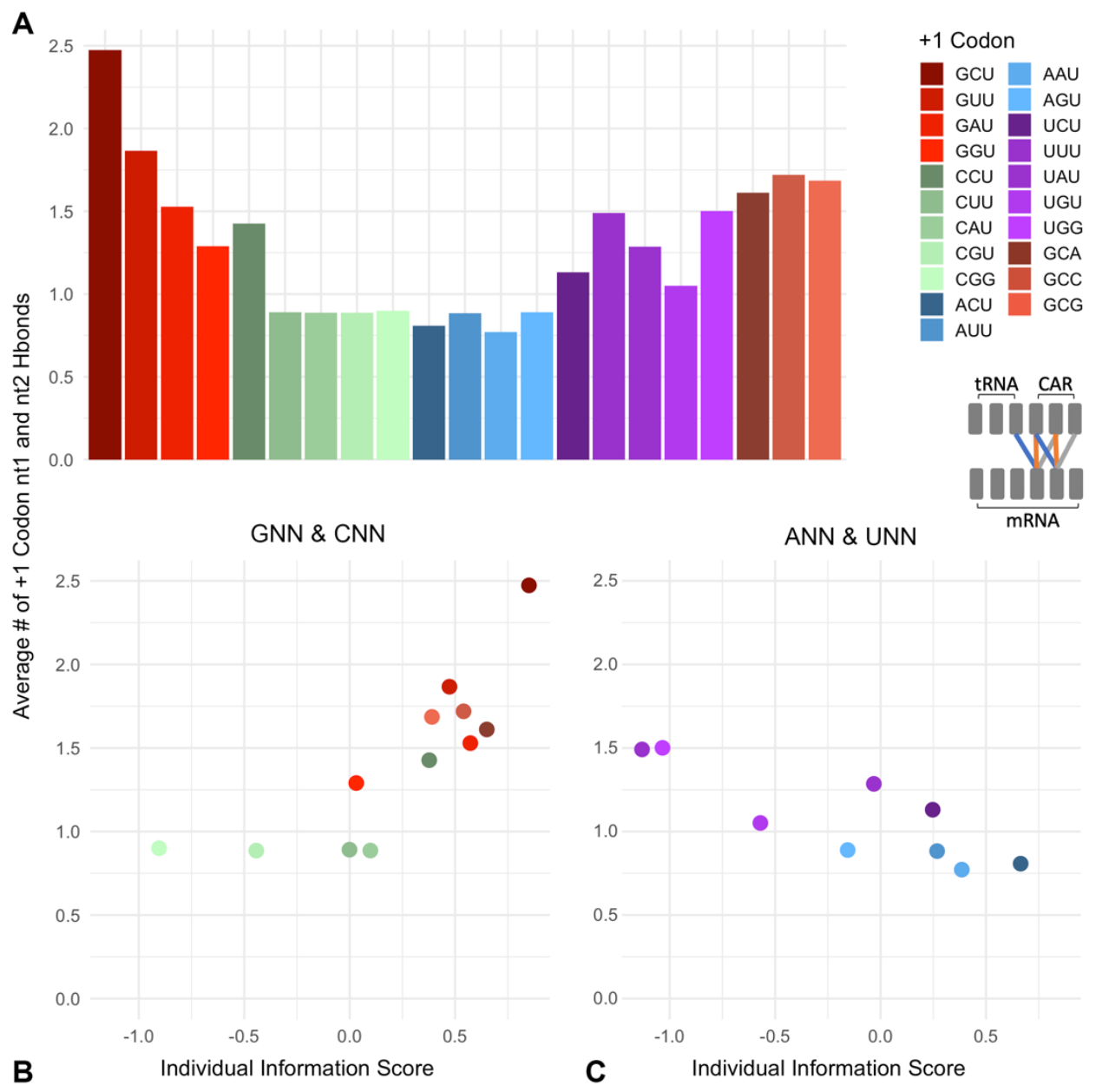

Figure 6. CAR interactions correlate with +1 codon individual information scores. (A) Summed $\mathrm{H}$-bond counts for positions 1 and 2 of +1 codons. (B) Comparison of these H-bond counts with individual information scores of $+1 \mathrm{GNN}$ and $+1 \mathrm{CNN}$ codons revealed a correlation (Spearman correlation coefficient $=0.818)$. $(\mathbf{C})$ However, +1 ANN and +1 UNN codons show a negative correlation (Spearman correlation coefficient $=-0.833$ ).

However, for codons with A1 (+1 ANU) which have low CAR H-bonding (Fig 3), or U1 (+1 UNU) which have incorrectly phased $\mathrm{H}$-bonding (Fig 4), we found a negative correlation of individual information scores of codons with the strength of their $\mathrm{CAR} \mathrm{H}$-bonding (correlation coefficient = -0.833; Fig 6C). Interestingly, ANU codons have individual information scores only a little below GNU (Fig 2B,C), suggesting that there may also be selection for codons (+1 ANU) that have consistent weak interactions with CAR. In contrast, UNU codons have some of the lowest individual information scores (Fig 2B,C), suggesting that there may be selection against the split or out-of-phase interactions of U1 with CAR (Fig 4). We speculate that this behavior could influence probabilities of frameshift events during translocation, and interestingly, $U$ at position 1 is consistent with the 7-nucleotide slippery sites associated with frameshifting events if the slippery site encompassed the A-site and +1 codons [33]. 
mRNA interactions modulate CAR integrity and anchoring

Stacking interactions can make important contributions to the stabilities of RNA structures [34]. In MD simulations of the $+1 \mathrm{GCU}$ ribosome subsystem, as discussed above, the CAR interaction surface is anchored to the tRNA nt34 through base stacking with C1054, and the CAR surface itself displays stacking interactions between its adjacent residues: base stacking between C1054/A1196 and cation-pi stacking between A1196/R146 [18,19]. We were curious whether +1 codons with weaker $\mathrm{H}$-bonding to CAR influenced the CAR anchoring or stacking interactions.
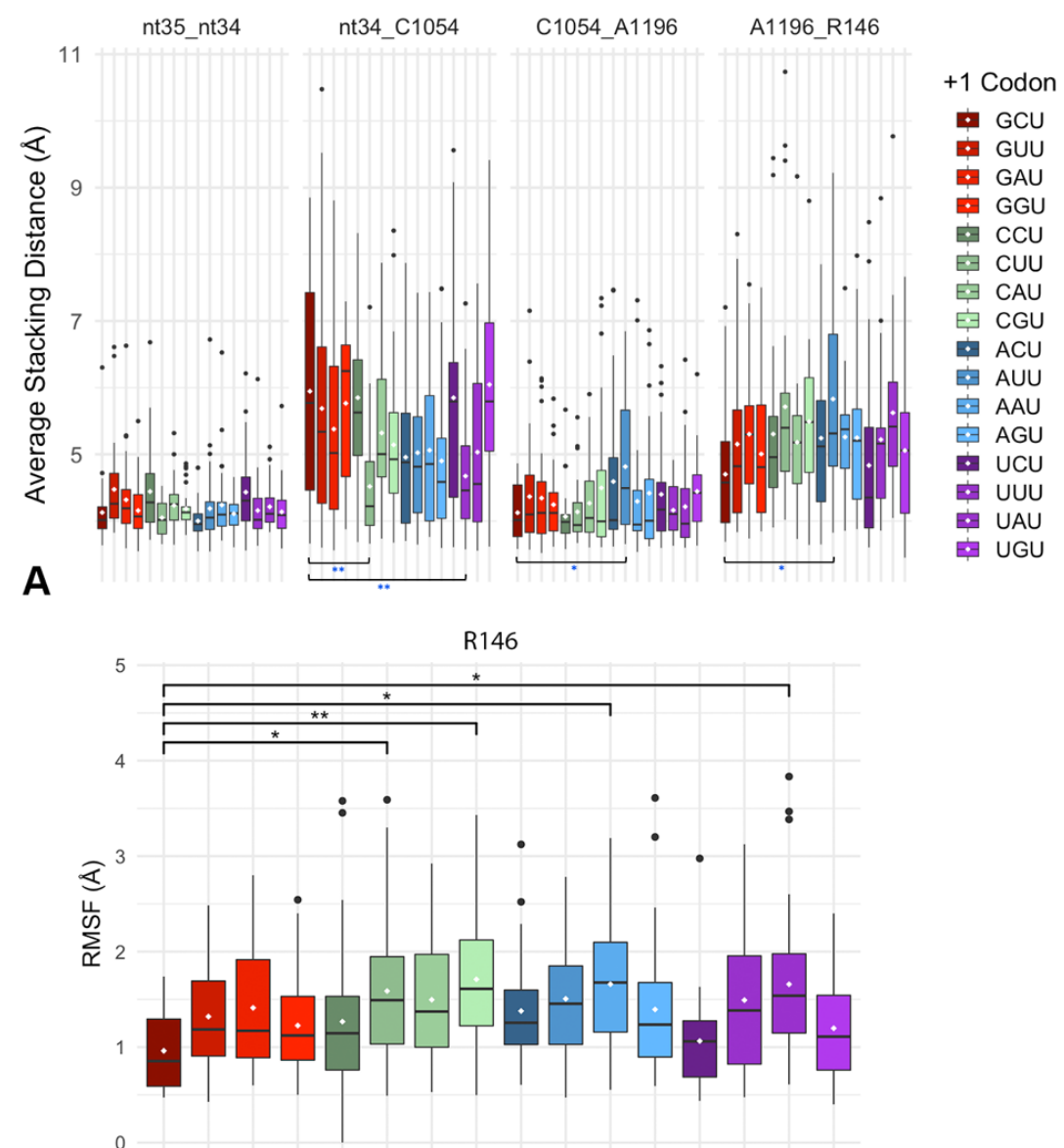

B

$$
0
$$

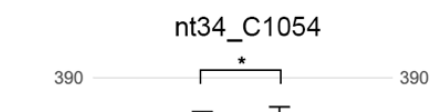

A1196_R146
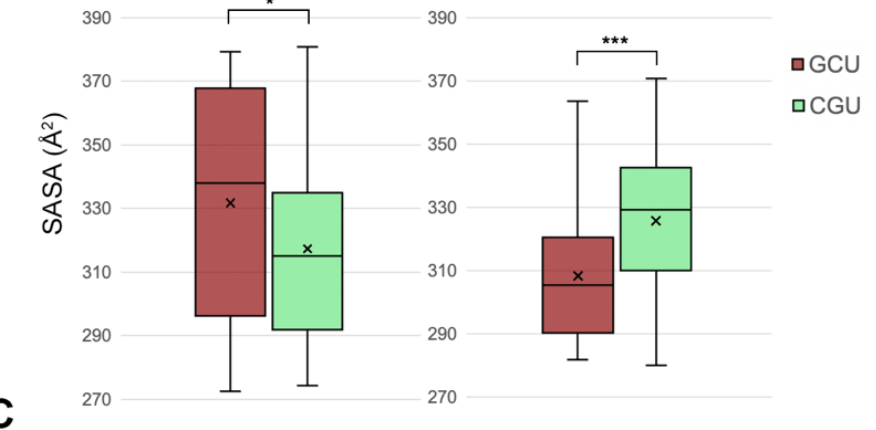

C

Figure 7. CAR stacking depends on +1 codons. (A) Stacking interactions in the CAR structure were assessed by measuring the center of mass (COM) distances between the base rings of C1054 and A1196, and between A1196 and the guanidinium group heavy atoms of R146. CAR anchoring was assessed through COM distance between C1054 and tRNA nt34. The optimal +1 GCU codon had the strongest A1196_R146 stacking and weakest nt34_C1054 anchoring. (B) RMSF of R146 was correspondingly lowest for +1 GCU. (C) Solvent accessible surface area (SASA) measurements 
comparing +1 GCU and +1 CGU codons similarly showed that +1 GCU has lower SASA for A1196_R146 reflecting its stronger stacking, and higher SASA for nt34_C1054 reflecting its weaker stacking.

Stacking between pairs of residues was analyzed by measuring the distance between the centers of mass (COM) of adjacent resides as described previously [18,19]. COM distances were calculated for four different stacking pairs: tRNA nt35 to nt34, tRNA nt34 to C1054, C1054 to A1196, and A1196 to R146. In general, COM distances between tRNA nt35 and nt34, and between $\mathrm{C} 1054$ and A1196, were not affected by the presence of different +1 codons (Fig 7A). However, the stacking interaction between tRNA nt34 and C1054 tended to be stronger (smaller COM distances; Fig 7A) for +1 codons that exhibit lower CAR/+1 codon $\mathrm{H}$-bonding (Fig 3 ). Indeed, +1 CUU and +1 UUU showed significantly lower COM distances compared to $+1 \mathrm{GCU}$, indicating improved base stacking. In contrast, the stacking interaction between A1196 and R146 tended to be less strong (higher COM distances; Fig 7A) for +1 codons that show weaker $\mathrm{CAR} /+1$ codon $\mathrm{H}$-bonding compared to $+1 \mathrm{GCU}$. Consistent with its poorer pi-cation stacking, R146 exhibited more variability in its positioning as revealed by its elevated RMSF (Root Mean Squared Fluctuations; Fig 7B). The RMSF measurements were made using the four core heavy atoms of the arginine guanidinium group ([18] and Fig S4). RMSF assessment of neighboring residues showed no significant differences when comparing the +1 GCU and +1 CGU subsystems (Fig S4).

We also measured solvent accessible surface area (SASA; $[18,35,36])$ between stacked residues comparing $+1 \mathrm{GCU}$ and $+1 \mathrm{CGU}$ as a test case (Fig 7C). The SASA measurement, comparing isolated stacked residue pairs, allowed us to assess the ability of water molecules to fit between the stacked residues. Compared to +1 GCU, +1 CGU showed greater SASA between R146 and A1196, confirming that their pi-cation stacking is less robust. In contrast, the +1 CGU subsystem showed reduced SASA between C1054 and tRNA nt34, indicating stronger anchoring of CAR.

In summary, these results showed that stronger $\mathrm{H}$-bond interactions between CAR and the +1 codon are associated with stronger stacking between A1196 and R146, suggesting that the $\mathrm{CAR} /+1$ codon interaction tends to stabilize the stacking interaction. However, in contrast, the CAR/+1 codon interaction tends to destabilize the anchoring of CAR to tRNA nt34, as evidenced by the weakened stacking between C1054 and tRNA nt34, suggesting that anchoring dominates when CAR is not as well held in place by interaction with the +1 codon.

\section{Different +1 codons exhibit different substates of the decoding center}

Our observations that different +1 codons have effects on $\mathrm{H}$-bonding between CAR and the +1 codon as well as the anchoring and stacking integrity of the CAR interface suggested that different +1 codon identities may lead to alternative dynamic substates of the ribosome decoding center. To investigate this further, we performed K-means clustering analysis [37] to determine whether different +1 codons are associated with different clusters representing alternative dynamic structures. 


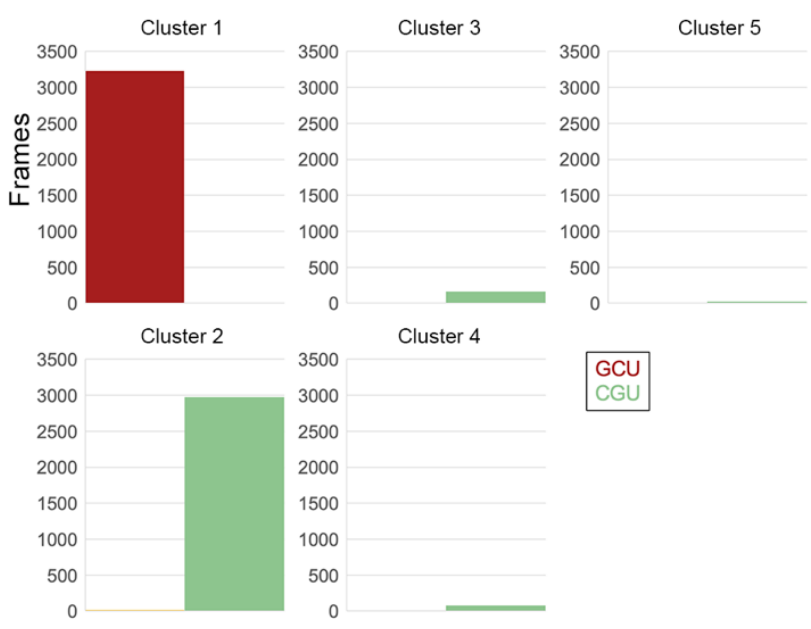

A. Unrestrained residues (backbone atoms) $\mathrm{K}=5$

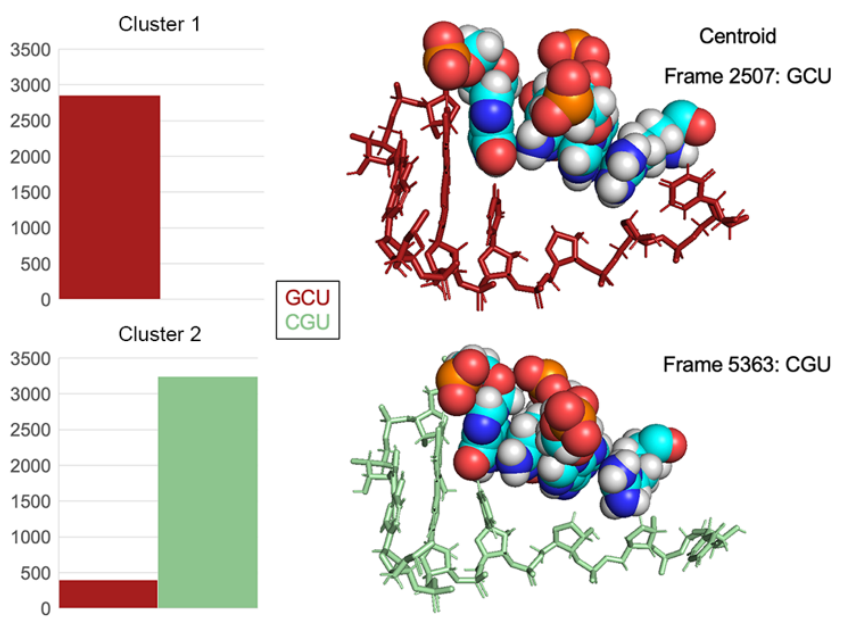

B. 12 residues (all atoms) $\mathrm{K}=2$

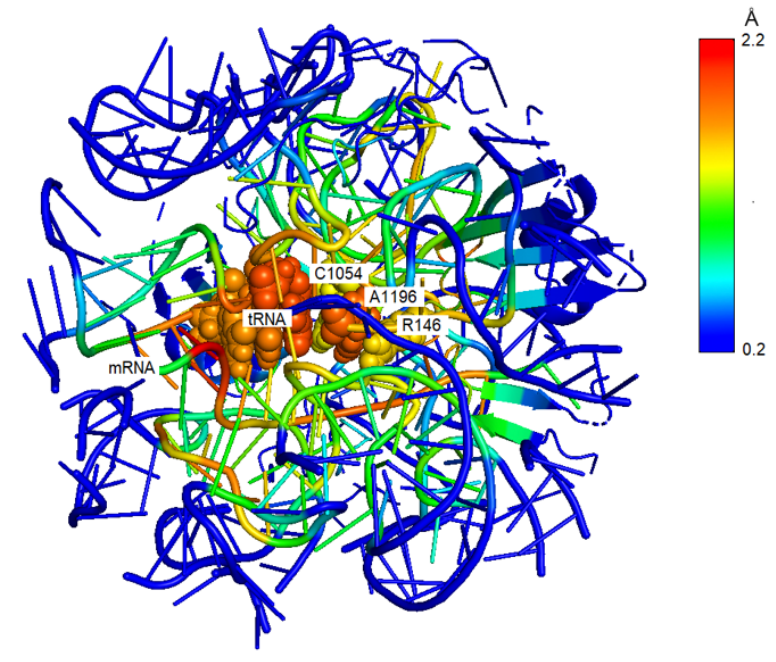

\section{Substate comparison}

Figure 8. The decoding center adopts different backbone structures depending on +1 codons. (A) K-means clustering was performed on concatenated trajectories for $+1 \mathrm{GCU}$ and $+1 \mathrm{CGU}$. Backbone atoms of the 321 unrestrained residues were used for clustering. Clustering with $\mathrm{K}=5$ revealed two dominant clusters, each populated by frames from only +1 GCU (cluster 1) or +1 CGU (cluster 2). $4.2 \%$ of $+1 \mathrm{CGU}$ frames were in other clusters. (B) K-means clustering was performed with all atoms of 12 residues (A-site codon and anticodon, +1 codon and CAR). Clustering with $\mathrm{K}=2$ 
revealed clusters dominated by $+1 \mathrm{GCU}$ or $+1 \mathrm{CGU}$. The centroid frame structures are illustrated using Van der Waals spheres for tRNA nt 34, C1054, A1196 and R146 (left to right). Cluster 1 (+1 GCU) shows weaker anchoring of CAR to tRNA nt 34 whereas the centroid for cluster 2 (+1 CGU) shows better CAR anchoring but poorer stacking of R146. The base rings of +1 codon nucleotides 1 and 2 are not illustrated and were not included in the clustering analysis. (C) Comparison of cluster 1 (GCU) and cluster 2 (CGU) from clustering with backbone atoms (panel A) revealed extensive differences throughout the subsystem neighborhood (reds and yellows in structure heat map). RMSF analysis was performed on trajectory frames from cluster 2 (4000 frames) using as a reference structure, the centroid from cluster 1 . As expected, the restrained onion shell residues show low RMSF values (blue).

For the K-means analysis, we compared $+1 \mathrm{GCU}$ and +1 CGU subsystems. 60 concatenated trajectories ( 30 for each +1 codon, 3200 frames total) were subjected to K-means clustering in which differences between aligned trajectory frames were computed using the summation of RMSD measurements of backbone atoms as the difference measure for clustering. Unexpectedly, setting $\mathrm{K}=5$ resulted in a complete separation of the $+1 \mathrm{GCU}$ and $+1 \mathrm{CGU}$ trajectories (Fig 8A), indicating that these two +1 codons are associated with distinct dynamic backbone substates that extend beyond the +1 codon. Increasing the number of clusters $(K=8)$ revealed additional minority clusters but left over $89 \%$ of the frames in the two dominant clusters. Decreasing the number of clusters $(K<5)$ led to a large cluster with over $95 \%$ of the frames from both dominant clusters of $\mathrm{K}=5$.

We carried out a second K-means analysis in which all atoms of only 12 residues-the A site and +1 codon, CAR and the tRNA anticodon-were used in the clustering steps. Again, the two dominant clusters $(K=2)$ showed almost complete separation of the GCU and CGU frames. The centroid structures for the two clusters represent the structures that are closest to all other structures in their respective clusters (Fig 8B). The centroid for GCU has poor anchoring of the CAR interface consistent with our earlier results. In contrast, the +1 CGU centroid shows good stacking alignment of C1054 with tRNA nt34 providing anchoring of CAR.

Comparison of the centroid structures for clusters 1 (+1 GCU) and 2 (+1 CGU) in Fig 8A showed that the differences between their two structures extend well beyond the CAR residues (Fig $8 \mathrm{C}$ ). This was demonstrated by performing RMSF analysis on the cluster 1 trajectories using the cluster 2 centroid as a reference structure. High RMSF values (reds and yellows in heat map; Fig $8 \mathrm{C}$ ) were detected throughout the subsystem of 321 unrestrained residues (inside the onion shell).

As summarized in Fig 3B, $+1 \mathrm{GCU}$ has the highest levels of $\mathrm{H}$-bonding between $\mathrm{CAR}$ and the +1 codon while $+1 \mathrm{CGU}$ is in a group of codons with the lowest $\mathrm{H}$-bonding levels. We expanded our K-means analysis to include +1 codons with intermediate levels of $\mathrm{H}$-bonding $(+1 \mathrm{CCU},+1$ GGU and +1 UGU) as well as +1 AAU which like +1 CGU has low H-bonding (Fig 9). 30 trajectories for each of the six +1 codons were concatenated for K-means analysis. Clustering using backbone atoms of the 321 unrestrained residues again revealed striking separation of high and intermediate interactors (+1 GCU, +1 CCU, +1 GGU and +1 UGU) from the low interactors (+1 CGU and +1 AAU). This indicates a strong preference of the ribosome subsystems for these two dominant yet distinctive backbone substates of the decoding center neighborhood. One substate is associated with CAR interaction with the +1 codon, and the other substate is associated with low interactions. Depending upon which codon has advanced to the +1 position during translocation (stage II), the decoding center would switch between these two substates. These results emphasize again the striking neighborhood effects of the +1 codon. 

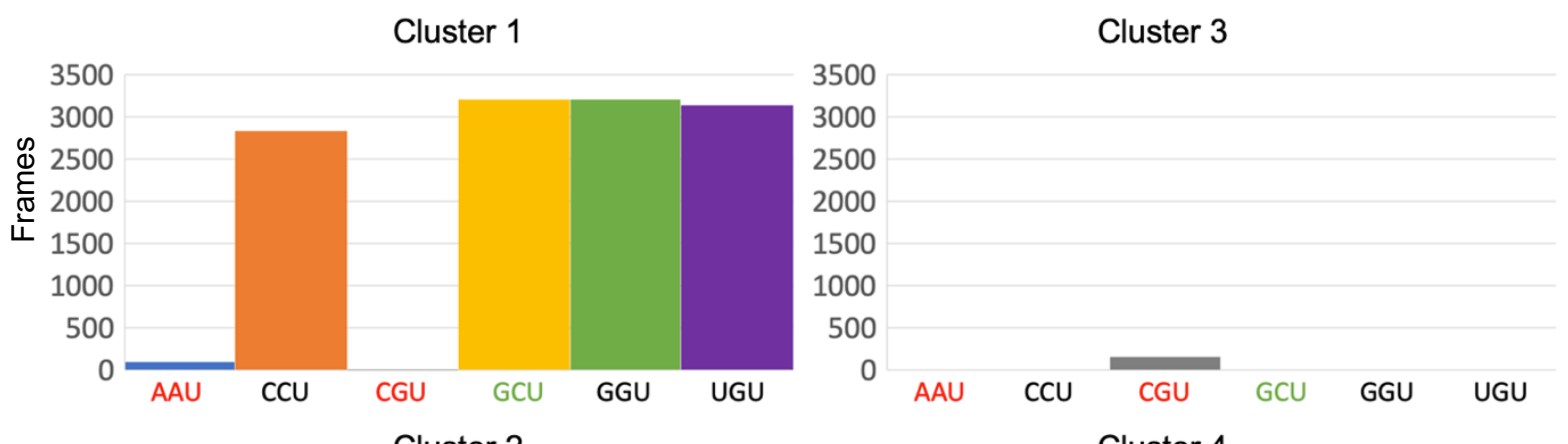

Cluster 2
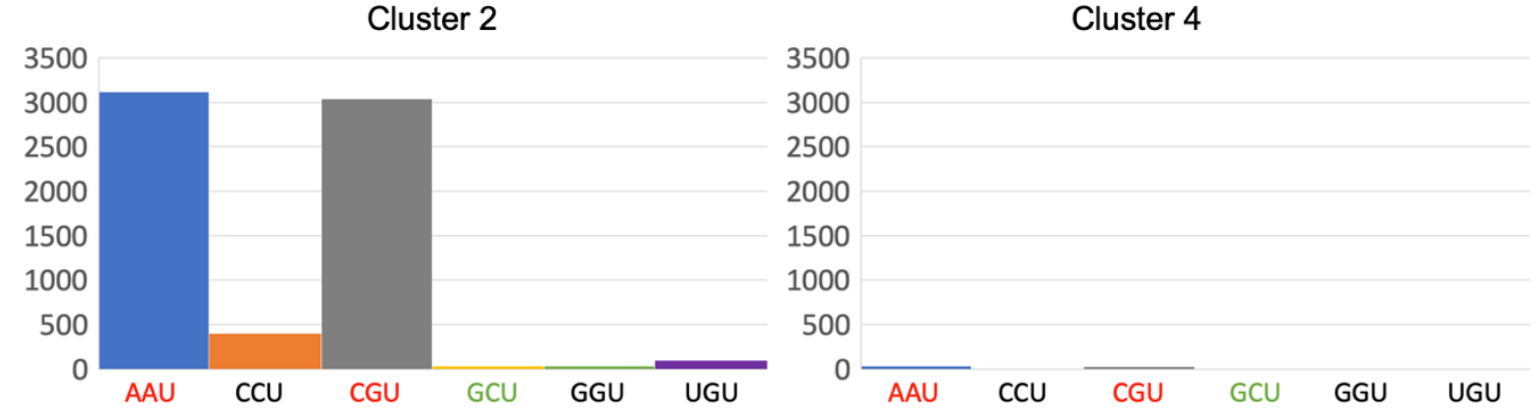

\section{A. Unrestrained residues (backbone) $\mathrm{K}=4$}
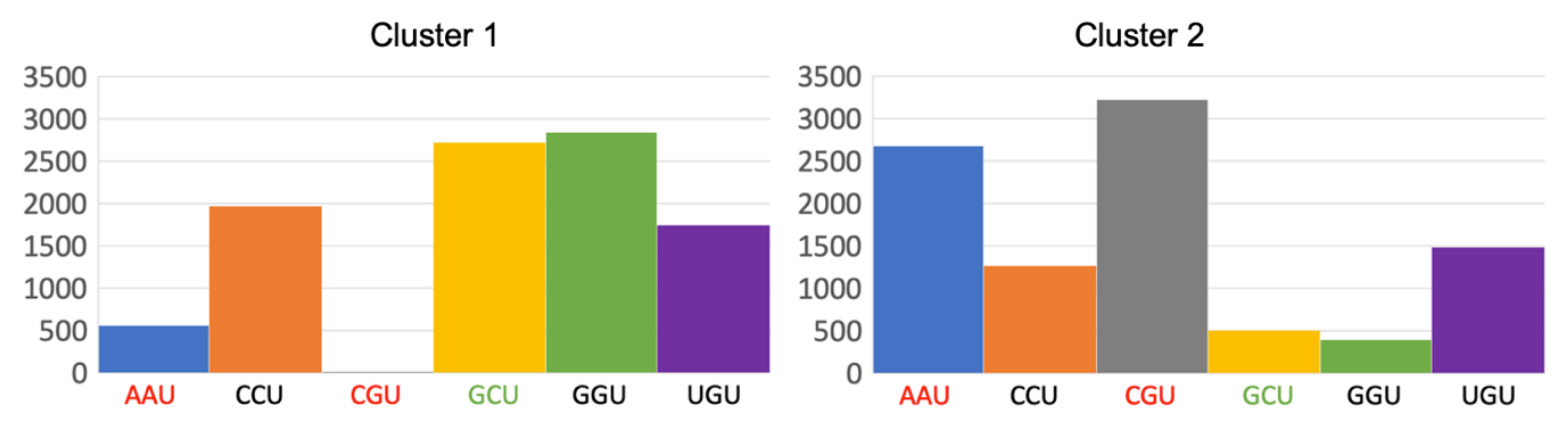

\section{B. 12 residues (all atom) $\mathrm{K}=2$}

Figure 9. Trajectories for six +1 codons were concatenated for $\mathrm{K}$-means analysis ( 30 trajectories each, 2 frames/ns). (A) Clustering $(K=4)$ with backbone atoms of unrestrained residues grouped together (cluster 1) frames for +1 codons with high (GCU) or intermediate (CCU, GGU, UGU) H-bonding between CAR and the +1 codon. Frames for +1 codons with low $\mathrm{CAR} /+1$ codon $\mathrm{H}$-bonding (AAU and CGU) were grouped in the another cluster (cluster 2), along with very small fractions of the CCU and UGU frames. (B) Clustering $(K=2)$ performed with all atoms of 12 residues (A-site codon and anticodon, +1 codon and CAR) revealed a cluster (cluster 1 ) dominated by GCU, GGU; the centroid frame for this cluster has weak anchoring of CAR to tRNA nt 34. The other cluster (cluster 2) is dominated by AAU and CGU, and its centroid frame (CGU, not shown) has good anchoring of CAR but weaker stacking of Rps3 R146.

\section{Conclusions}

In this study, we have shown that the identity of the +1 codon, the codon next to enter the ribosome $A$ site, has a pronounced effect on the behavior of the decoding center structure and dynamics, an effect that is driven by whether or not the +1 codon interacts with the CAR interface, a surface that shows sequence-specific interactions with the +1 codon. CAR exhibits a strong preference for $+1 \mathrm{GCN}$ codons, resulting in switch-like behavior of the ribosome subsystem depending on whether or not the codon conforms to GCN. The consequences of GCN codon interactions with CAR on protein translation remain to be elucidated, although 
preliminary indirect observations suggest that energetically favorable CAR/+1 codon interactions with initial codons of protein ORFs (ramp regions) may be particularly influential in modulating protein translation under certain cellular conditions such as stress when CAR is hypothesized to be functional [17-19]. It is interesting to speculate that over evolutionary timescales, selection for GCN codons in the ramp regions of ORF may have rendered genes sensitive to CAR-mediated regulation. GCN codes for alanine, and the small non-polar R group of alanine may be expected to often have fairly neutral effects on the structure and function of evolving proteins if selection for GCN led to incorporation of alanine residues into the protein structure.

\section{Methods}

Information theoretic analysis of codons

Python scripts were used to analyze yeast ORF sequences of 6033 verified and uncharacterized genes (downloaded May 2021; https://www.yeastgenome.org). For calculations of individual information scores [26], background nucleotide frequencies were calculated for all ORFs (A: 0.328, C: 0.192, G: 0.204, T: 0.276). Individual information scores were calculated using a position weight matrix based on a meta-codon for superimposed codons $3,4,5,6$ and 7 (see Fig 2).

\section{Molecular dynamics analysis}

Using the AMBER 18 suite [31], MD simulations were performed with a 494-residue subsystem of the ribosome centered around C1054 of the CAR surface as described previously [18] except where noted below. A harmonic potential force $\left(20 \mathrm{kcal} / \mathrm{mol} \AA^{2}\right)$ was applied to the residues at the surface of the subsystem (the onion shell) in order to retain the translocation stage II structure (PDB ID 5JUP; [20]). The subsystem, based on cryo-EM structure 5JUP, includes parts of a viral IRES that mimics the tRNA and mRNA.

Editing of +1 codon nucleotides was accomplished by removing atoms not shared with the replacement nucleotide. AMBER's tLEaP was then used to "grow in" missing atoms for the new residue based on a standard geometry. Each tested +1 codon was characterized using 30 independent MD trajectories (twenty 60-ns trajectories, and ten 100-ns trajectories). Each sample MD run was initiated with the same energy-minimized structure which became an independent experimental run with random velocity assignment during heating, followed by equilibration before production MD. RMSD deviations of RNA and protein backbone atoms were monitored to ensure that the subsystem was stable, typically after 20 ns (Fig S1), and our subsequent analysis of the trajectories did not include these initial $20 \mathrm{~ns}$.

$\mathrm{H}$-bonding between CAR and the +1 codon as well as $\mathrm{A}$-site codon/anticodon interactions were characterized using Python scripts to parse the results of AMBER's cpptraj [38] hbond function (out and avgout outputs) which returns $\mathrm{H}$-bonds with an acceptor-to-donor heavy atom distance less than $3.0 \AA$ and an angle cutoff of $135^{\circ}$.

A1196 stacking with C1054 (or R146) was characterized using the AMBER cpptraj distance function to measure the distances between the center-of-mass of the A1196 base with the C1054 base (or the R146 guanidinium group). Anchoring of C1054 to tRNA nt 34 through stacking was similarly measured. We also monitored water accessibility between stacked groups by measuring the solvent accessible surface area (SASA) surrounding the two bases, or the guanidinium group and base, using the fast LCPO algorithm [36] computed by the surf function in AMBER cpptraj. The geometries of base stacking interactions were also visualized using a polar coordinate representation as described by Bottaro et al. [32] to examine a single stacking pair of bases over multiple frames of trajectories-revealing centering of the stacked bases $(\rho)$ and relative rotations of the base rings $(\theta)$. 
Root mean square fluctuation (RMSF) was measured using the AMBER cpptraj rmsf function on the base rings of RNA nucleotides and the guanidinium group of R146 as described [18].

\section{Clustering analysis of trajectories}

K-means clustering was performed as described [37] using a concatenation of trajectories with different +1 codons. All frames from the concatenated trajectories were assigned to the cluster with their closest centroid structure based on an RMSD measurement using the backbone atoms of all unrestrained residues in our subsystem (not including onion shell residues). Nonbackbone atoms of nucleotides 1 and 2 of the +1 codon were removed allowing a single parameter-topology (parmtop) file to be used for the trajectories with different +1 codons. Kmeans analysis was also performed using all atoms of 12 residues (A-site codon and anticodon, +1 codon and CAR). The resulting centroids provided representative structures for each cluster. These structures were viewed using Pymol [39] and virtual molecular dynamics (VMD; [40,41]). To assess differences between structures in two clusters of interest, we performing RMSF on residues in one of the clusters using, as a reference structure, the centroid for the second comparison cluster. The RMSF values were visualized on the centroid structure of the first cluster by editing the B-factor column of its pdf file and visualizing in Pymol.

Acknowledgments: We thank David Beveridge, David Langley, Ishita Mukerji, Colin Smith, Joe Coolon, Jack Kwon, Jungwoo Cho, Abdel Elsayed, and Audrey McMahon for discussions, and Henk Meij for technical assistance with high-performance computing.

Supplementary Materials: Figure S1: RMSD profiles for trajectories; Figure S2: H-bonding of CAR with +1 NCU codons; Figure S3: Influences of nucleotide 3 of the +1 codon; Figure S4: RMSF comparison of $+1 \mathrm{GCU}$ and +1 CGU trajectories.

\section{References}

1. Pavitt, G.D. Regulation of translation initiation factor elF2B at the hub of the integrated stress response. WIREs RNA 2018, 9, e1491.

2. $\quad$ Sanchez, M.; Lin, Y.; Yang, C.; McQuary, P.; Campos, A.R.; Blanc, P.A.; Wolf, D.A. Cross Talk between elF2a and eEF2 Phosphorylation Pathways Optimizes Translational Arrest in Response to Oxidative Stress. iScience 2019, 20, 466-480.

3. Shenton, D.; Smirnova, J.B.; Selley, J.N.; Carroll, K.; Hubbard, S.J.; Pavitt, G.D.; Ashe, M.P.; Grant, C.M. Global Translational Responses to Oxidative Stress Impact upon Multiple Levels of Protein Synthesis. J Biol Chem 2006, 281, 29011-29021.

4. Chan, C.T.; Dyavaiah, M.; DeMott, M.S.; Taghizadeh, K.; Dedon, P.C.; Begley, T.J. A quantitative systems approach reveals dynamic control of tRNA modifications during cellular stress. PLoS genetics 2010, 6, e1001247, doi:10.1371/journal.pgen.1001247. PMC3002981

5. $\quad \mathrm{Gu}, \mathrm{C}$.; Begley, T.J.; Dedon, P.C. tRNA modifications regulate translation during cellular stress. FEBS Lett 2014, 588, 4287-4296, doi:10.1016/j.febslet.2014.09.038. PMC4403629

6. Hou, Y.M.; Gamper, H.; Yang, W. Post-transcriptional modifications to tRNA--a response to the genetic code degeneracy. RNA 2015, 21, 642-644, doi:10.1261/rna.049825.115. PMC4371315

7. Schosserer, M.; Nadege Minois; Angerer, T.B.; Amring, M.; Dellago, H.; Harreither, E.; CallePerez, A.; Pircher, A.; Gerstl, M.P.; Pfeifenberger, S., et al. Methylation of ribosomal RNA by NSUN5 is a conserved mechanism modulating organismal lifespan. Nat Commun 2015, 6, 6158.

8. Sloan, K.E.; Warda, A.S.; Sharma, S.; Entian, K.D.; Lafontaine, D.L.J.; Bohnsack, M.T. Tuning the ribosome: The influence of rRNA modification on eukaryotic ribosome biogenesis and function. RNA Biology 2017, 14, 1138-1152.

9. Zhao, B.S.; Roundtree, I.A.; He, C. Post-transcriptional gene regulationby mRNA modifications. Nat Rev Mol Cell Biol 2017, 18, 31-42.

10. Back, S.; Gorman, A.W.; Vogel, C.; Silva, G.M. Site-Specific K63 Ubiquitinomics Provides Insights into Translation Regulation under Stress. J Proteome Res 2019, 18, 309-318.

11. Silva, G.M.; Daniel; Finley, D.; Vogel, C. K63 polyubiquitination is a new modulator of the oxidative stress response. Nature structural \& molecular biology 2015, 22. 
12. Farley-Barnes, K.I.; McCann, K.L.; Ogawa, L.M.; Merkel, J.; Surovtseva, Y.V.; Baserga, S.J. Diverse Regulators of Human Ribosome Biogenesis Discovered by Changes in Nucleolar Number. Cell Rep 2018, 22, 1923-1934, doi:10.1016/j.celrep.2018.01.056. PMC5828527

13. Genuth, N.R.; Barna, M. The Discovery of Ribosome Heterogeneity and Its Implications for Gene Regulation and Organismal Life. Mol Cell 2018, 71, 364-374, doi:10.1016/j.molcel.2018.07.018.

PMC6092941

14. Monaco, P.L.; Marcel, V.; Diaz, J.J.; Catez, F. 2'-O-Methylation of Ribosomal RNA: Towards an Epitranscriptomic Control of Translation? Biomolecules 2018, 8, doi:10.3390/biom8040106. PMC6316387

15. Shi, Z.; Fujii, K.; Kovary, K.M.; Genuth, N.R.; Rost, H.L.; Teruel, M.N.; Barna, M. Heterogeneous Ribosomes Preferentially Translate Distinct Subpools of mRNAs Genome-wide. Mol Cell 2017, 67, 71-83 e77, doi:10.1016/j.molcel.2017.05.021. PMC5548184

16. Xue, S.; Barna, M. Specialized ribosomes: a new frontier in gene regulation and organismal biology. Nat Rev Mol Cell Biol 2012, 13, 355-369, doi:10.1038/nrm3359. PMC4039366

17. Barr, W.A.; Sheth, R.B.; Kwon, J.; Cho, J.; Glickman, J.W.; Hart, F.; Chatterji, O.K.; Scopino, K.; Voelkel-Meiman, K.; Krizanc, D., et al. GCN sensitive protein translation in yeast. PloS one 2020, 15, e0233197. PMC7500604

18. Scopino, K.; Dalgarno, C.; Nachmanoff, C.; Krizanc, D.; Thayer, K.M.; Weir, M.P. Arginine Methylation Regulates Ribosome CAR Function. Int J Mol Sci 2021, 22, 1335. PMC7866298

19. Scopino, K.; Williams, E.; Elsayed, A.; Barr, W.A.; Krizanc, D.; Thayer, K.M.; Weir, M.P. A Ribosome Interaction Surface Sensitive to mRNA GCN Periodicity Biomolecules 2020, 10 , doi:10.3390/biom10060849. PMC7357141

20. Abeyrathne, P.D.; Koh, C.S.; Grant, T.; Grigorieff, N.; Korostelev, A.A. Ensemble cryo-EM uncovers inchworm-like translocation of a viral IRES through the ribosome. Elife 2016, 5, doi:10.7554/eLife.14874. PMC4896748

21. Lv, F.; Zhang, T.; Zhou, Z.; Gao, S.; Wong, C.C.; Zhou, J.Q.; Ding, J. Structural basis for Sfm1 functioning as a protein arginine methyltransferase. Cell Discov 2015, 1, 15037, doi:10.1038/celldisc.2015.37. PMC4860837

22. Young, B.D.; Weiss, D.I.; Zurita-Lopez, C.I.; Webb, K.J.; Clarke, S.G.; McBride, A.E. Identification of methylated proteins in the yeast small ribosomal subunit: a role for SPOUT methyltransferases in protein arginine methylation. Biochemistry 2012, 51, 5091-5104, doi:10.1021/bi300186g.

PMC3383884

23. Gerashchenko, M.V.; Gladyshev, V.N. Translation inhibitors cause abnormalities in ribosome profiling experiments. Nucleic Acids Res 2014, 42, e134, doi:10.1093/nar/gku671. PMC4176156

24. Crawford, R.A.; Pavitt, G.D. Translational regulation in response to stress in Saccharomyces cerevisiae. Yeast 2019, 36, 5-21.

25. Tuller, T.; Carmi, A.; Vestsigian, K.; Navon, S.; Dorfan, Y.; Zaborske, J.; Pan, T.; Dahan, O.; Furman, I.; Pilpel, Y. An evolutionarily conserved mechanism for controlling the efficiency of protein translation. Cell 2010, 141, 344-354, doi:S0092-8674(10)00319-3 [pii] 10.1016/j.cell.2010.03.031.

26. Schneider, T.D. Information content of individual genetic sequences. J Theor Biol 1997, 189, 427441.

27. Shultzaberger, R.K.; Roberts, L.R.; Lyakhov, I.G.; Sidorov, I.A.; Stephen, A.G.; Fisher, R.J.; Schneider, T.D. Correlation between binding rate constants and individual information of E. coli Fis binding sites. Nucleic Acids Res 2007, 35, 5275-5282.

28. Sharp, P.M.; Li, W.H. The codon Adaptation Index--a measure of directional synonymous codon usage bias, and its potential applications. Nucleic Acids Res 1987, 15, 1281-1295.

29. Lagunez-Otero, J.; Trifonov, E.N. mRNA periodical infrastructure complementary to the proofreading site in the ribosome. Journal of biomolecular structure \& dynamics 1992, 10, 455-464, doi:10.1080/07391102.1992.10508662.

30. Mendoza, L.; Mondragon, M.; Lagunez-Otero, J. Interaction of the 530 ribosomal site with regions of mRNA. Biosystems 1998, 46, 293-298.

31. Case, D.A.; Ben-Shalom, I.Y.; Brozell, S.R.; Cerutti, D.S.; Cheatham, I., T.E. ; Cruzeiro, V.W.D.; Darden, T.A.; Duke, R.E.; Ghoreishi, D.; Gilson, M.K., et al. AMBER 18. 2018.

32. Bottaro, S.; Di Palma, F.; Bussi, G. The role of nucleobase interactions in RNA structure and dynamics. Nucleic Acids Res 2014, 42, 13306-13314, doi:10.1093/nar/gku972. PMC4245972

33. Jacobs, J.L.; Belew, A.T.; Rakauskaite, R.; Dinman, J.D. Identification of functional, endogenous programmed -1 ribosomal frameshift signals in the genome of Saccharomyces cerevisiae. Nucleic Acids Res 2007, 35, 165-174. 
34. Sponer, J.; Sponer, J.E.; Mladek, A.; Jurecka, P.; Banas, P.; Otyepka, M. Nature and magnitude of aromatic base stacking in DNA and RNA: Quantum chemistry, molecular mechanics, and experiment. Biopolymers 2013, 99, 978-988, doi:10.1002/bip.22322.

35. Amaro, R.E.; Cheng, X.; Ivanov, I.; Xu, D.; McCammon, J.A. Characterizing loop dynamics and ligand recognition in human- and avian-type influenza neuraminidases via generalized born molecular dynamics and end-point free energy calculations. J Am Chem Soc 2009, 131, $4702-$ 4709, doi:10.1021/ja8085643. PMC2665887

36. Weiser, J.; Shenkin, P.; Still, W. Approximate atomic surfaces from linear combinations of pairwise overlaps (LCPO). J Comput Chem 1999, 20, 217-230.

37. Thayer, K.M.; Lakhani, B.; Beveridge, D.L. Molecular Dynamics-Markov State Model of Protein Ligand Binding and Allostery in CRIB-PDZ: Conformational Selection and Induced Fit. J Phys Chem B 2017, 121, 5509-5514, doi:10.1021/acs.jpcb.7b02083.

38. Roe, D.R.; Cheatham III, T.E. PTRAJ and CPPTRAJ: software for processing and analysis of molecular synamics trajectory data. J Chem Theory Comput 2013, 9, 3084-3095.

39. DeLano, W.L. The PyMOL Molecular Graphics System.; New York, 2010.

40. Hsin, J.; Arkhipov, A.; Yin, Y.; Stone, J.E.; Schulten, K. Using VMD: An introductory tutorial. Current Protocols in Bioinformatics 2008, 14.

41. Humphrey, W.; Dalke, A.; Schulten, K. VMD: Visual Molecular Dynamics. . J. Molec Graph 1986, $14,33-38$. 